\title{
Visualization of the HSV-1 Portal Complex in situ by cryo-electron tomography
}

\author{
G. Cardone, ${ }^{*}$ D. Winkler, ${ }^{*}$ B.L. Trus**, W. W. Newcomb,*** J. C. Brown, ${ }^{* * *}$ A. C. Steven* \\ *Laboratory of Structural Biology, NIAMS, and ** Imaging Sciences Laboratory, CIT, National \\ Institutes of Health, DHHS, Bethesda, MD, USA. \\ *** Department of Microbiology and Cancer Center, University of Virginia Health System, \\ Charlottesville, VA, USA.
}

Herpes simplex virus type 1 (HSV-1) consists of a icosahedral nucleocapsid, surrounded by a proteinaceous tegument and the viral envelope [1]. Its assembly begins with the formation of a spherical procapsid, which transforms into the mature capsid through a major conformational change [2]. In infected cells, capsid maturation is accompanied by packaging of DNA into the capsid via the portal channel, the UL6 gene product [3]. As with double-stranded DNA bacteriophages, the HSV-1 portal is situated at a unique vertex, the other eleven being occupied by pentamers of the major capsid protein. Cryo-EM of UL6 molecules obtained by baculovirus expression revealed a predominant dodecameric structure, with an axial channel and a marked asymmetry from end-to-end [4]. However, it is still not known how the portal is inserted into the capsid shell, nor whether it undergoes any conformational change during capsid maturation.

To address these questions, we are studying isolated A-capsids (empty, mature capsids) by cryoelectron tomography. Seven tilt series at $-4 \mu \mathrm{m}$ defocus, angular increment $1^{\circ}$, and average angular range $\left[-57^{\circ},+57^{\circ}\right]$, were acquired. From the resulting tomograms (e.g. Fig. 1), 150 capsids were extracted. The best (in-plane) resolution for individual capsids is 55-60 Å by NLOO2D [5]. At this resolution, and also because of the anisotropy generated by the missing-wedge effect, we found that the portal vertex could not be reliably discriminated from the other eleven by visual criteria. Accordingly, we developed a protocol based on three-dimensional classification and correlation averaging. Each vertex capsomer was excised from each capsid, and converted to a two-dimensional representation by taking a transverse axial section through the vertex after rotational averaging around that axis. The rotation was performed in the Fourier domain, in order to exclude from the average the spatial frequencies contained in the missing wedge. The vertices were then classified in terms of a model-based correlation analysis, using four models obtained by implanting the cryoEM model of the portal on either the inner surface of the outer surface and in both orientations. For a given capsid, the vertex with the highest correlation was assigned as the portal vertex. The subtomograms containing these vertices were then subjected to several rounds of three-dimenaional correlation averaging. They yield an image that tallies with the portal mounted on the outer surface of the capsid floor, with its narrow end pointing outwards (Fig. 2a). As a control, the averaged vertices selected by the wrong models gave an image consistent with the major capsid protein pentamer (e.g. Fig. 2b).

\section{References}

[1] K. Grünewald et al., Science 302 (2003) 1396.

[2] J. B. Heymann et al., Nature Struct. Biol. 10 (2003) 334.

[3] W. W. Newcomb et al., J. Virol. 79 (2005) 10540.

[4] B. L. Trus et al., J. Virol. 78 (2004) 12668.

[5] G. Cardone et al., J. Struct. Biol. 151 (2005) 117. 


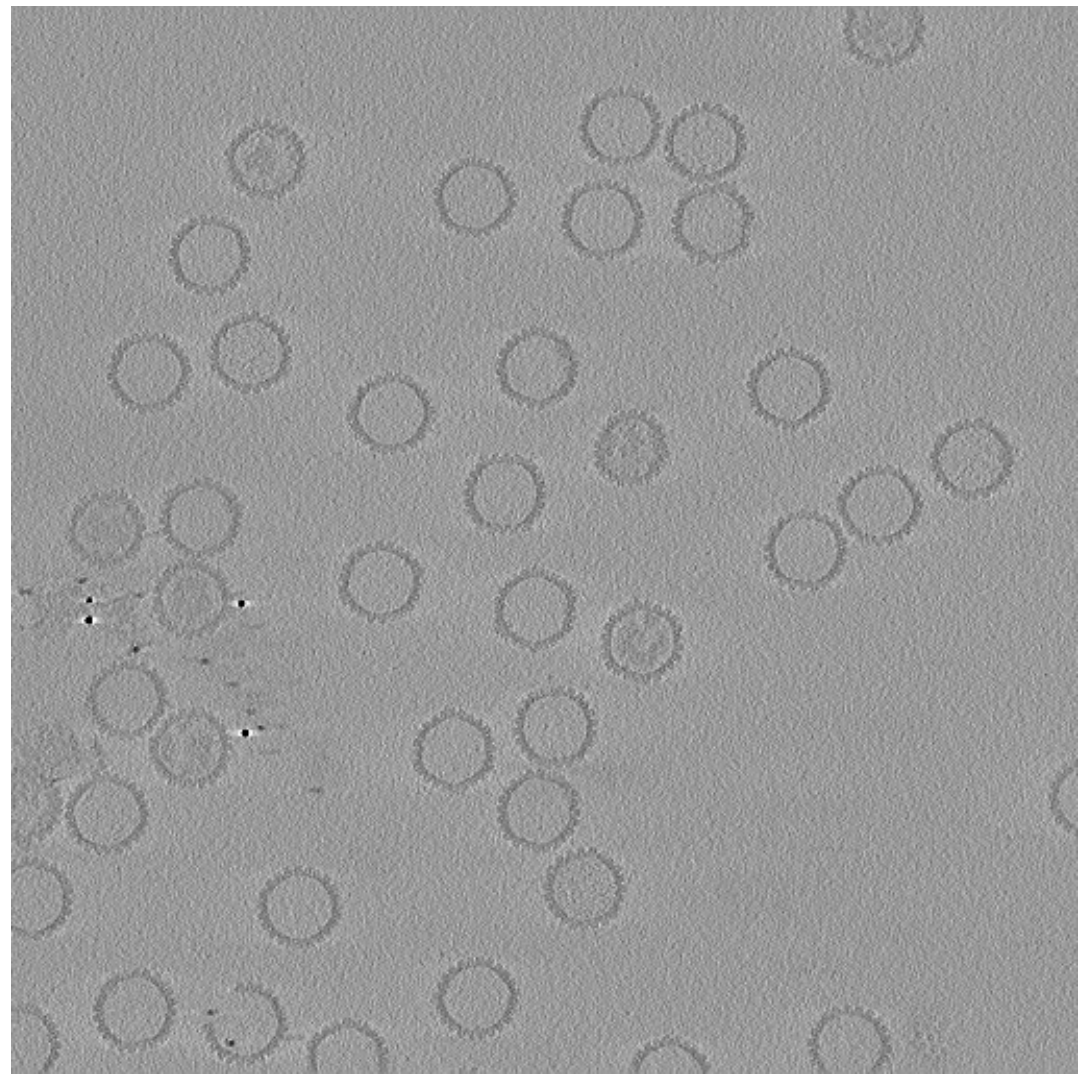

Fig. 1. An x-y section through a tomographic reconstruction of HSV-1 A-capsids. The section is 1.5 $\mathrm{nm}$ thick (no denoising was applied). The capsids are $125 \mathrm{~nm}$ in diameter.

a)
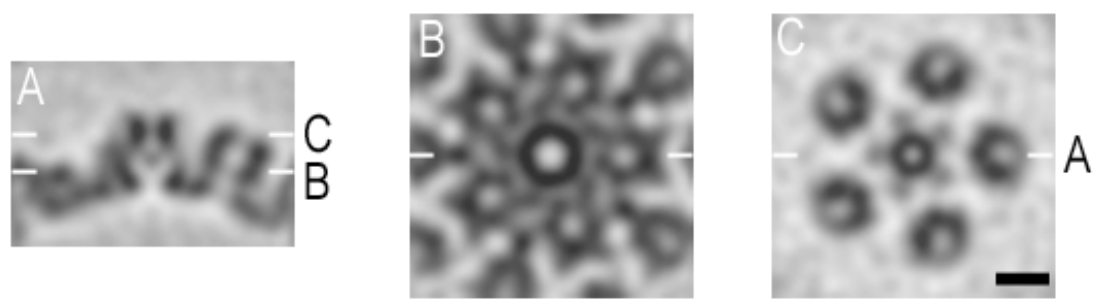

b)
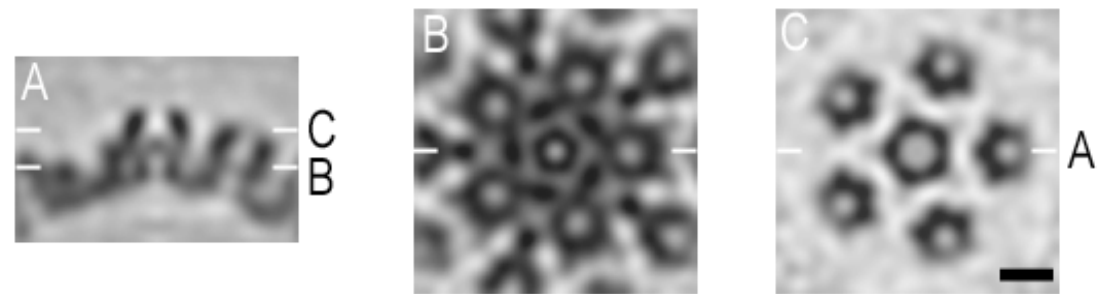

Fig. 2. a) Axial central sections through averaged sub-tomograms, each containing an A-capsid vertex and the surrounding five pentamers. (a) Vertices classified as containing the portal; b) Control of vertices selected by an erroneous classification model have a pentamer of the major capsid protein. Planes $\mathrm{B}$ and $\mathrm{C}$ are perpendicular to plane $\mathrm{A}$, and the relative locations of these planes are marked on the figures. Bar $10 \mathrm{~nm}$. 